\title{
EXPLORANDO LAS MOTIVACIONES DE LOS FUTUROS DOCENTES DE EDUCACIÓN PRIMARIA EN LA ELECCIÓN DE SUS ESTUDIOS
}

\author{
Exploring the Motivations of Future Primary \\ School Teachers in their Career Choices
}

Miguel Pérez-Ferra ${ }^{1}$

mperez@ujaen.es

José Manuel Martos-Ortega ${ }^{2}$

josemanuel.martos@unir.net

Rocío Quijano-López ${ }^{1}$

rquijano@ujaen.es

Inmaculada García-Martínez ${ }^{1}$

igmartin@ujaen.es

${ }^{1}$ Universidad de Jaén. España

${ }^{2}$ Universidad Internacional de La Rioja. España

Recibido: 21/09/2020

Revisado: 2/11/2020

Aprobado: 19/12/2020

Preprint: 13/05/2021

Publicado: 15/07/2021

\section{Resumen}

Inmersos en una crisis identitaria en la que la profesión docente ha sido devaluada con el paso del tiempo, es momento de replantear las razones que motivan a los aspirantes a maestro a convertirse en futuros docentes. La finalidad de este estudio es analizar los factores que determinaron su elección profesional. Se seleccionaron 38 autobiografías, atendiendo a la profundidad de las reflexiones de los estudiantes. Se utilizó la metodología de análisis de relatos autobiográficos a través de la "teoría fundamentada". Los resultados mostraron motivaciones intrínsecas y extrínsecas. Entre las primeras se identificaron las siguientes: el sentido de compromiso de la educación, y el interés para trabajar con nińos y contribuir a desarrollar su proyecto personal de vida y las recompensas morales ligadas a esta actividad profesional; entre las motivaciones extrínsecas se destacaron las buenas condiciones laborales y el amplio tiempo de vacaciones, así como la influencia de padres y familiares maestros, la admiración por algunos profesores que tuvieron y experiencias de voluntariado durante sus estudios de secundaria. Este estudio avanza hacia un mejor entendimiento de la identidad profesional de los aspirantes a convertirse en maestros de educación primaria.

Palabras clave: elección de la carrera, identidad profesional, maestros en formación, motivación.

\section{Abstract}

Faced with an identity crisis where the teaching profession has been undervalued over time, it is time to reconsider the reasons that encourage students to become future teachers. The aim of this study is to analyse the factors that determined their career choice. Thirty-eight autobiographies were selected, taking into account the depth of the students' reflections. The methodology used was the analysis of autobiographical narratives through "Grounded Theory". The results differentiated between intrinsic and extrinsic motivations. Among the first ones were identified: their commitment to education, their interest in working with children and contributing to their personal life project, and the moral rewards linked to this professional activity; among the extrinsic motivations, good working conditions, or extended holiday time were highlighted. Likewise, the impact of parents and family teachers, their admiration for some teachers who had or volunteer experiences during their secondary school studies were also factors that contributed to this choice. This research advances towards a better understanding of the preservice teacher's professional identity.

Keywords: career choice, motivation, preservice teachers, professional identity. 


\section{Introducción y revisión de la literatura}

La calidad de los procesos de enseñanza-aprendizaje está determinada fundamentalmente por el compromiso ético, el desarrollo de la identidad profesional docente y, como determinante de los dos anteriores, la motivación que ha orientado a un estudiante del título de maestro de Educación Primaria a elegir esa carrera, aspecto en el que se pretende profundizar en el presente trabajo.

La motivación constituye una fuerza que aporta determinación y dirige el comportamiento humano del maestro (Feu et al., 2016). La motivación intrínseca está esencialmente unida a la vocación, entendida como la asunción de un compromiso con el "otro", el estudiante, en el sentido de transcender a uno mismo (Orbegoso, 2016) o bien como el descubrimiento que hace el estudiante sobre en qué consiste su ser y cómo debe interaccionar con el mundo (Ayerra, 2016). Es la motivación intrínseca la que permite que se mantenga esa acción vocacional en el tiempo, entendida como parte de la llamada actitud proactiva (Ossa et al., 2018).

La motivación intrínseca queda definida por el sentido de acogida que supone la educación mediante la vocación de servicio, las recompensas morales por el trabajo bien hecho, la posibilidad de educar y formar a los demás, etc. Lo cierto es que sin motivación intrínseca no es posible el desarrollo de una futura identidad profesional docente, sobre todo en un espacio de tiempo en el que se da un tránsito de la identidad como estudiantes a su entidad como profesores (Breijo \& Fernández, 2020). Sin embargo, la motivación extrínseca supone el desempeño de una actividad profesional por el logro de recompensas externas. Es la motivación de aquellos estudiantes que eligen la carrera por influencia de los padres o familiares, de profesores que fueron modelo para ellos, por las buenas condiciones laborales o la experiencia de trabajo con niños mediante acciones de voluntariado, entre otros aspectos (Del Valle-Uzcátegui, 2019).

La idiosincrasia que encierra la profesión de maestro permite conjugar ambos tipos de motivación. Los estudiantes pueden elegir la profesión de maestro, entre otros motivos, porque tienen vocación de servicio y altruismo, motivación intrínseca, pero dicha motivación puede haber surgido por el hecho de conocer las vivencias de un miembro de su familia en el ejercicio de la profesión y percibir que compartían esos sentimientos o percepciones (Gratacós, 2014).

Las argumentaciones precedentes abren un espacio adecuado para afirmar que la motivación extrínseca, en algunas ocasiones, tiene un efecto incoativo respecto a la intrínseca, que es la que define y consolida el compromiso profesional. Se alude a la motivación contextual, extrínseca, entendida como la capacidad de sentir atracción por una actividad humana que tiene consecuencias en el desarrollo de la motivación interna y está definida por factores sociales, familiares y de estabilidad, que actúan de catalizadores para el desarrollo de la motivación interna (Núñez \& León, 2018).

En esta línea de pensamiento se ha manifestado Gómez-Acuñas (2003) al afirmar que las "variables tales como el afecto positivo, la disposición optimista y la autoestima están relacionadas con la motivación intrínseca” (p. 162). Sin embargo, resulta paradójico que, a pesar de la cantidad de investigaciones que se han llevado a cabo sobre el tema, en el proceso de selección de los estudiantes que quieren estudiar para ser maestros no se han considerado los aspectos motivacionales (Egido, 2010).

Se han llevado a cabo estudios con jóvenes pertenecientes a la clase media y notas elevadas de acceso a la universidad, en los que predominan las motivaciones intrínsecas, mientras que en aquellos pertenecientes a sectores sociales menos favorecidos prevalecen las motivaciones extrínsecas (Aisenson et al., 2010).

Una de las evidencias derivadas de la presente revisión bibliográfica que conviene destacar es que las dimensiones que definen la identidad profesional docente son vitales en este proceso electivo. Nos referimos a la dimensión existencial, definida por las vivencias que aportan las historias de vida de los estudiantes, que se deben completar o deconstruir, si fuese el caso, a través de la reflexión sobre sus 
narrativas autobiográficas (Jarauta-Barranco, 2017), ya que suponen el modo de ver y de verse frente a sí.

Lo esencial de la identidad profesional, contextualizada en un espacio sociocultural, suele ser el centro educativo, la familia o el círculo de amigos, que orientan la elección de los estudiantes, fundamentalmente durante el desarrollo de sus estudios de secundaria (Cortés et al., 2014; Ferreira-Bolognani \& Mendes-Nacarato, 2015). Identidad que debe transcender los planteamientos político-económicos y orientarse a una propuesta ético-pedagógica en la que "el centro de la formación es el reconocimiento, respeto y acogida al otro" (Mínguez-Vallejos, 2019, p. 13).

Las argumentaciones precedentes conducen al siguiente problema de investigación: "Qué motivaciones determinan que los estudiantes elijan la carrera de maestro", que se concreta en los siguientes objetivos: a) analizar las reflexiones que realizan los estudiantes durante sus relatos autobiográficos y ver si fueron determinantes para la elección de sus estudios, b) conocer en qué sentido se orientan las autobiografías respecto a las motivaciones que han incidido en la elección de la carrera.

\section{Método}

La construcción de la identidad personal no se puede abordar sin considerar la historia personal que determina las motivaciones que están en el origen de la elección de una titulación. Las narraciones autobiográficas son reveladoras en la medida que descubren el camino recorrido y la génesis de los proyectos de vida de las personas, ordenan las experiencias, vivencias y sentimientos que antes de ser narrados pueden parecer inconexos (Dubard \& Nicourd, 2017; González \& Padilla, 2014), y evidencian la riqueza de significados que se construyen, necesarios para comprender y entender el mundo que rodea a una persona ( $\mathrm{Ba}-$ rriga et al., 2018). Es decir, remiten a una de las dimensiones constitutivas de la experiencia: "la capacidad del ser humano para configurar narrativamente su existencia y para biografiar su experiencia singular del mundo histórico y social" (Delory-Momberger, 2015a, 2015b). Desde esta perspectiva, la persona tiene la posibilidad de reconstruir su trayectoria personal, tematizando sobre la misma e interpretándola (Runge \& Muñoz, 2015).

Se ha utilizado el relato autobiográfico de los estudiantes de primer curso del grado de maestro en Educación Primaria porque adscribe lo narrado a un contexto escolar, familiar y social y a una realidad ideológica (Clemens \& Tierney, 2020), ámbitos en los que se enmarca la existencia de los informantes y las dimensiones determinantes a la hora de decidir la elección profesional y su formación universitaria (Leite et al., 2019). Son realidades que suponen un continuum en el proceso de construcción de su identidad profesional (Watson \& Mcluckie, 2020). En efecto, las narrativas autobiográficas son relatos escritos en primera persona sobre una serie de experiencias vitales relativas al objeto de estudio específico. En ellas se produce una simbiosis entre narrador y protagonista, es decir, plasman retrospectivamente por escrito su propia experiencia, y seleccionan los acontecimientos que le parecen relevantes para explicarlos, reinterpretarlos y transmitir el significado que tienen para él a partir de un proceso de autorreflexión (Moriña, 2017).

La metodología seguida se articuló en tres fases: la recogida de los datos, su análisis, y su reducción e interpretación (Ballestín \& Fábregas, 2018a; Bernal, 2016; Flick, 2012; Packer, 2018).

En la primera fase se invitó a los alumnos de primer curso del grado de Maestro en Educación Primaria a construir sus narrativas autobiográficas, y se seleccionaron 38. El procedimiento de selección estuvo determinado por el nivel de profundización alcanzado en los aspectos que responden a la temática y objetivos de la investigación. En suma, fueron las autobiografías que presentaban mayor variedad de datos, con relatos bien definidos de las experiencias que fueron determinantes para optar por los estudios del título de grado de Educación Primaria.

Posteriormente, desde la perspectiva de un enfoque ético de la investigación, se solicitó la autorización a los estudiantes para utilizar sus autobiografías en 
la investigación, por medio de un consentimiento informado (Moriña, 2017), firmado por los autores y los investigadores, en el que se explican distintos aspectos (tema de investigación, instrumentos de recogida de datos, duración del estudio, etc.) y que explicita el derecho de los participantes a saber que van a ser estudiados, la naturaleza de la investigación y el impacto de los resultados.

La segunda fase se centró en la categorización y análisis de las autobiografías de los estudiantes seleccionados. El proceso seguido consistió en analizar cada narrativa autobiográfica y construir nodos temáticos mediante una codificación abierta (Carrero et al., 2012), siguiendo los procesos inductivos propios de la teoría fundamentada (Ballestín, 2018b; Rosenthal, 2018) y utilizando el software de análisis cualitativo NVivo11. A partir de la codificación abierta se identificaron un conjunto de unidades de análisis que emergen de los datos y describen acontecimientos, propiedades y relaciones significativas para nuestro objeto de estudio. Las unidades de análisis se agruparon en diez categorías que reducen los datos y se establecieron propiedades y dimensiones significativas para el objeto de estudio (Andreu et al., 2007): 1) dimensión vocacional, 2) influencia de los padres y familiares, 3) formar y educar a los demás, 4) buenas condiciones laborales, 5) influencia del profesorado, 6) experiencia de trabajo con niños y voluntariado,
7) vocación de servicio y altruismo, 8) recompensas morales, 9) interacción con los niños, 10) influencia de los amigos. Dichas categorías se aglutinaron en torno a dos metacategorías: motivaciones intrínsecas y motivaciones extrínsecas (Ver Tabla 1).

La tercera fase se centró en comprender, a partir de los datos, los resultados del análisis, estableciendo conexiones e inferencias situadas (Murcio, 2013). Las dos familias de categorías han sido fundamentales para conocer e interpretar el pensamiento de los estudiantes noveles sobre las razones de su elección de la carrera de maestro, razones que se reflejan en el presente informe de investigación. El informe de investigación se redactó haciendo evidentes las voces de los estudiantes mediante sus relatos autobiográficos, atendiendo a un sentido ético de la investigación en el que la voz de los estudiantes no se diluya en el discurso de los investigadores (Thomas \& Arden, 2008).

\section{Resultados}

El análisis e interpretación de los relatos autobiográficos de los alumnos, atendiendo a la matriz de codificación, dibuja dos grandes bloques de motivaciones para elegir los estudios de Magisterio, a saber: motivaciones extrínsecas y motivaciones intrínsecas (Ver Tabla 1).

Tabla 1. Matriz de codificación: motivación intrínseca y extrínseca

\begin{tabular}{lclc}
\hline Motivaciones intrínsecas & $\begin{array}{c}\text { N. }{ }^{\circ} \text { de ref. de } \\
\text { codificación }\end{array}$ & \multicolumn{1}{c}{ Motivaciones extrínsecas } & $\begin{array}{c}\mathbf{N} \text {. }^{\circ} \text { de ref. de } \\
\text { codificación }\end{array}$ \\
\hline Dimensión vocacional & 19 & Influencia de los padres y familiares & 12 \\
\hline Formar y educar a los demás & 12 & Buenas condiciones laborales & 11 \\
\hline Vocación de servicio y altruismo & 5 & Influencia del profesorado & 9 \\
\hline Recompensas morales & 4 & $\begin{array}{l}\text { Experiencia de trabajo con niños y } \\
\text { voluntariado }\end{array}$ & 8 \\
\hline Interacción con los niños & 4 & Influencia de los amigos & 2 \\
\hline & & & $\mathbf{4 1}$ \\
\hline Total de referencias & $\mathbf{4 4}$ & Total de referencias & \\
\hline
\end{tabular}

Nota: Elaboración propia. 


\subsection{Motivaciones intrínsecas}

\subsubsection{Dimensión vocacional}

Entre las motivaciones intrínsecas sobresale la vocacional. Los alumnos y alumnas hablan de su admiración e identificación con la carrera. En las narrativas se señala que desde pequeños les ha gustado trabajar como maestro o maestra. (Nota: todas las citas que no tienen identificación de autoría corresponden a testimonios de estudiantes que mantenemos anónimos).

Sinceramente, una de las razones que me han llevado a matricularme en el grado de maestro en Educación Primaria es porque desde pequeña me ha gustado trabajar como maestra. Para mí ser maestra es algo más que enseñar a unos nińos lo que tienen que aprender, cómo tienen que aprender... Para mí esta profesión significa algo más, ya que a partir de ella voy a poder ayudar a los nińos en todo lo que necesiten y todo lo que esté en mis manos, porque me encanta todo lo relacionado con los niños.

Este interés por la profesión se mostraba en los juegos desde una edad temprana.

En primer lugar por vocación, ya que el trabajo de maestro es la profesión que desde pequeño me ha gustado e incluso he jugado en mi infancia.

Es cierto que, aunque me gusta mucho esta profesión, mi familia también me ha influido un poco en mi decisión, ya que ellos ven este grado como una profesión a partir de la cual yo puedo disfrutar trabajando, porque saben que desde pequeña me divertía jugando a los maestros con otros niños, enseñando cosas... Por eso creo que no me voy a equivocar en la decisión que he tomado.

Al describir dicha dimensión vocacional, con frecuencia se evoca una imagen idílica y de admiración del maestro y de su tarea docente que lleva a calificarla como un "sueño".

Para terminar esta narrativa, decir que desde pequeña sońaba con ser maestra y a día de hoy solo estoy a un paso de poder conseguir mi sueño. No tiraré la toalla, lucharé porque el día de mañana quiero ser yo la que imparta conocimiento y sabiduría a niños con ganas de aprender, de crecer y de vivir.
Dicha idealización y admiración están unidas a los referentes familiares, principalmente los padres, o los maestros que tuvieron en su infancia.

Mi madre es maestra de infantil y siempre he admirado su profesión y he querido seguir sus pasos. $Y$ desde que era una nińa tenía claro que me quería dedicar a la enseñanza.

Mi padre ha sido el que me ha transmitido las sensaciones que se sienten al estar en contacto con los niños en un aula en la que se desarrolla el proceso de enseñanza-aprendizaje. Disfruto con la ternura y el cariño que ofrecen los nińos, ver cómo se desarrollan y maduran psicomotrizmente.

La admiración lleva consigo el reconocimiento de poseer un conjunto de cualidades y actitudes que facilitarán la tarea docente.

\subsubsection{Interés por la formación y la educación de las personas}

Otro conjunto de motivaciones se vincula al interés por la formación y educación de personas.

El interés por la enseñanza, en efecto, constituye un dinamizador para la elección de la titulación en la que busca "formarse para ser un maestro competente, de esos que nunca se olvidan”. Enseñar a los demás constituye un valor fundamental para la sociedad y la persona, ya que se ofrecen aprendizajes esenciales y básicos necesarios para la vida. Para esta labor educativa es imprescindible la figura del maestro.

Me he decantado por los estudios de Grado en Educación Primaria porque desde siempre la educación me ha parecido algo fundamental. En esta tarea la figura del maestro es clave, pues es una ayuda en el aprendizaje. Además, siempre he admirado esta labor, ya que es de vital importancia para nuestro desarrollo como personas.

En las narrativas se vincula la tarea del maestro con su contribución al desarrollo personal de los alumnos, a la construcción de su identidad como personas, estableciendo las bases de futuros aprendizajes.

Pienso que el ser maestro será muy gratificante, ya que me dedicaré a enseñar y educar a niños. Gracias 
a mi trabajo en la escuela ellos podrán continuar

formándose para unos futuros estudios superiores.

Desde esta perspectiva reconocen su futura tarea como una responsabilidad por el poder de influencia que tienen en la vida del alumnado por medio de la transmisión y desarrollo de valores.

Considero la enseñanza el pilar de todo lo demás, donde se asientan todos los demás recursos de un país, sobre todo su cultura. Apostar por la enseñanza en un país es la mejor apuesta posible, ya que si esta es buena, todo lo demás lo será. Pienso que es una de las profesiones más bonitas que existen, ya que dedicas tu vida a enseñar e inculcar una serie de principios y valores a los niños que dentro de un futuro serán el futuro de nuestro país.

\subsubsection{Vocación de servicio y altruismo (ayudar a los demás)}

La elección de la titulación está vinculada a la dimensión altruista y de servicio a los demás como una experiencia compartida, principalmente, por las personas de su entorno familiar que trabajan en el ámbito de la enseñanza o vivida desde su trayectoria escolar.

Tengo familiares que se han dedicado a la enseñanza y siempre que hemos hablado de su profesión la han descrito como un trabajo gratificante, ya que ayudas a otras personas. También tuve la suerte de que muchos de los maestros que he tenido han sido muy cercanos y me han ayudado en todo lo que han podido. Gracias a ellos aprendí con los libros, pero también me ayudaron a formarme como persona dentro y fuera del colegio.

En las narrativas se insiste, junto con las dimensiones vinculadas al aprendizaje, en la importancia de ayudar a los demás en su desarrollo como personas.

\subsubsection{Recompensas morales}

Existe un conjunto de narrativas que inciden en las recompensas no materiales, es decir, morales, que le aportará la práctica de la futura profesión docente, ya que le permitirá "disfrutar trabajando", ser maestro es un "trabajo gratificante".

\subsubsection{Interacción con los niños}

El interés por los procesos de interacción que acontecen en el ámbito escolar es otra de las dimensiones que determinan la elección de la carrera. Dicha interacción constituye un contexto propicio para el aprendizaje del futuro maestro, ya que "el mismo, junto a sus alumnos, aprenden juntos”.

\subsection{Motivaciones extrínsecas}

\subsubsection{Influencia de los padres y los familiares que se dedican a la enseńanza (12 referencias codificadas)}

En los relatos se hace referencia a los padres; a otros familiares: tíos, primos y hermanos; y a su pareja como un factor influyente para la elección de la carrera.

En el contexto familiar, los padres y demás miembros de la familia que se dedican a la enseñanza comparten experiencias y anécdotas vinculadas a su práctica docente.

También otra razón por la que he escogido estudiar el Grado en Educación Primaria viene influenciada por miembros de mi familia que se dedican también a la enseńanza y cuando nos juntamos en las comidas familiares me encanta escucharlos, sobre todo cuando cuentan anécdotas de sus alumnos.

Por medio de la narración de las experiencias que describen las vivencias y las emociones que comparten en el aula junto con los alumnos en el contexto de los procesos de enseñanza-aprendizaje han conseguido transmitir "la admiración por la profesión". Les anima la ilusión que ven en sus familiares próximos que comentan anécdotas y aspectos de su trabajo docente:

[...] veo en mi madre un efecto gratificante en ese trabajo que beneficia a otras personas.

[...] la ilusión que se refleja en sus rostros, no puedo evitar sentir cierta envidia y querer estar en el lugar de ellos y realizar una profesión tan bonita como la de maestro.

Esta dimensión gratificante del trabajo docente pesa más que "el salario, el horario o el tipo de trabajo". 
Por tanto, los estudiantes afirman en los fragmentos de las narrativas, que presenta la matriz de codificación como significativa, una admiración por la satisfacción que muestran sus padres en el ejercicio docente como determinante de la construcción de la identidad personal y el desarrollo profesional.

\subsubsection{Buenas condiciones laborales}

La búsqueda de unas buenas condiciones laborales, la estabilidad laboral por el hecho de ser funcionario y tener una buena calidad de vida será una motivación que determine la elección de la carrera.

[...] debo decir que también me motiva la búsqueda de una mejor calidad de vida, una comodidad, ya que he trabajado en la construcción, y ahora he decidido retomar mis estudios, terminar mi carrera y sacarme una oposición, un puesto de trabajo fijo con mis vacaciones y todos los beneficios que tiene un funcionario.

Por otra parte, para algún alumno que tiene un origen social modesto, ya que proviene de contextos familiares con rentas no muy altas, la elección de la carrera se ve como una forma de tener estabilidad económica.

En este país, trabajar como docente (generalmente) permite tener un nivel de vida medio, algo que llama bastante la atención. Por supuesto que, si no es algo que me atrajese, nunca me podría dedicar a ello, pero sí ayuda saber, cuando se proviene de un seno familiar humilde, que vas a poder tener una vida de nivel medio.

$\mathrm{Al}$ caracterizar las condiciones que posibilitan la calidad del trabajo y tener un buen nivel de vida sobresale el tema del salario, ya que es un trabajo "bien remunerado" y en el contexto actual "está mejor pagado que otro tipo de trabajos en los que dedicando más horas cobran bastante menos de lo que recibe un maestro". Junto con el salario, los estudiantes valoran el horario laboral, que permite "tener libre casi todas las tardes y los fines de semana", para tener tiempo para el ocio o la familia.

Junto con los aspectos relacionados con el salario y el horario laboral, sobresale el tema de las vacaciones, que le hace preguntarse a un informante: “¿A quién no le gustaría tener dos meses de vacaciones y encima cobrando?".

Por último, un informante señala el hecho de que la profesión constituye un trabajo fácil, que no exige esfuerzo aparentemente, elemento que pesa más que el salario, el horario o las mismas vacaciones.

\subsubsection{Influencia del profesorado}

Una tercera categoría alude a la incidencia que han podido ejercer maestros y profesores en la toma de decisiones a la hora de elegir la titulación.

[...] personalmente, durante mi etapa educativa he conocido a varios profesores que ejercían su trabajo realmente por vocación. En concreto, en Bachillerato tuve un profesor de latín excepcional que fue de gran influencia para mí porque me aportó muchísimos conocimientos y también a ver la vida desde distintos puntos de vista.

A los alumnos y alumnas les llama la atención la forma en la que los profesores dinamizaban los procesos de enseñanza-aprendizaje.

Esta es una de las razones por las que yo me decidí a estudiar esta carrera, por una profesora que tuve en la primaria, la cual era maravillosa, conseguía que las clases fuesen amenas, divertidas y que te gustase el hecho de estar sentado o sentada seis horas al día en clase, gracias a ella descubrí que la enseñanza puede ser algo divertido si uno se lo propone...

Para mí la gran influencia por la que he elegido la enseñanza es un profesor de matemáticas, química y física que además era psicólogo, ese hombre me ha ayudado mucho, ya que era mi profesor de clases particulares, además de ser profesor de instituto. Él ha sido el responsable de que yo, hoy día, pueda estar estudiando en la universidad, la verdad que es un profesor fantástico y me ayudaba mucho con sus consejos y sus explicaciones que para mí son las mejores, no hay nadie que explique matemáticas, física y química como Juan. Las explicaciones de este hombre eran de verdad una maravilla y hasta el más distraído en clase, después de clases de apoyo de Juan, se enteraba perfectamente. 
Eran apasionados de su profesión, disfrutaban con la enseñanza y la implicación con su trabajo.

Otro profesor que me marcó mucho fue el de Filosofía, por supuesto, al principio no lo soportábamos, pero al mes, más o menos, no podíamos dejar de dar filosofía y a todo el mundo le gustaba. Las paradojas de la vida, era el más exigente y con el que más disfrutábamos de la filosofía, veíamos que era un apasionado de lo que daba y así lo enseñaba.

Por último, señalan la cercanía de los maestros de su etapa escolar con los alumnos.

La elección la tomé tras hablar con una gran persona como es Diego Maroto, director de Salesianos San Agustín (Linares), que además es un buen amigo, acompañante personal y mi padrino, él me aconsejó que, conociéndome, sabiendo mis actitudes e inquietudes, era la mejor elección y la que seguro no me voy a arrepentir. Esto mismo me lo ha dicho mucha otra gente después.

A pesar de ser conscientes todos los alumnos del respeto que le debíamos profesar por la condición de ser el maestro, él se empeñaba constantemente en romper esa barrera y ser uno más. Como conclusión, añadir que tengo la total certeza y seguridad de que esta es la profesión que debo y quiero realizar.

Es sabido que en la influencia que ejercen los profesores sobre los estudiantes hay una admiración por el aspecto vocacional y la consiguiente inserción de aspectos motivacionales, sin embargo, hay que decir que en los fragmentos de las autobiografías que se han considerado no se alude a circunstancias diferentes a la mera actividad instructiva o al adecuado proceder de los docentes para que la docencia sea agradable y formativa para los estudiantes, pues siendo ello necesario, no es suficiente para considerar y determinar lo que es la enseńanza.

\subsubsection{Experiencias de trabajo con niños y volun- tariado}

Los alumnos manifiestan que ya conocen la acción docente y, por tanto, no constituye una novedad para ellos, ya que han tenido experiencias previas de trabajo educativo con niños en distintos contextos, principalmente el familiar y el del voluntariado y la catequesis.

- Familiar

El ámbito familiar constituye un contexto propicio para realizar distintas prácticas de enseñanza. El contacto con hermanos y primos de menor edad ha permitido ayudar en sus tareas escolares, situaciones que han despertado el interés por el mundo educativo o de la enseńanza, y han sido un acicate para elegir estudiar la carrera de maestro.

La segunda razón por la que me decidí a estudiar esta carrera es porque me gusta estar con niños pequeños, tengo primos y hermanos pequeños y desde siempre me ha gustado el ayudarles a aprender a leer, con las tablas de multiplicar, etc.

Este tipo de actividades de apoyo en las labores escolares les ha aportado a los informantes experimentar el interés de los niños por el aprendizaje, al contemplar "los ojos despiertos que reflejan la sorpresa ante un nuevo desafío", y ha reclamado de ellos respuestas creativas y empáticas "para resolver cualquier situación o problemática intelectual al hilo de la realización de los deberes".

Al redactar estas narrativas, y al realizar una mirada retrospectiva, los informantes consideran este apoyo a familiares en las tareas escolares un incidente crítico que les ha marcado profundamente en la elección de la carrera de maestro.

- Voluntariado y catequesis

Junto con el ámbito familiar, muchos de los informantes han entrado en contacto con el ámbito educativo no formal en contextos de voluntariado con niños y jóvenes y de actividades de formación religiosa o catequesis.

Todo esto de dar clase no es nuevo para mí, ya que desde hace años soy parte de un grupo de animadores salesianos que nos formamos como animadores socioculturales y de tiempo libre, con lo cual ya tengo algún curso realizado relacionado con la educación. Cada viernes me enfrento a un grupo de chavales a los que intento educar siguiendo el sistema preventivo de Don Bosco. 
He estado en contacto con niños y niñas porque he sido catequista y soy monitora, desde hace tres años, de actividades con adolescentes en mi antiguo colegio. Me hace mucha ilusión trabajar con ellos y me gustaría en un futuro marcarles de forma positiva como me marcaron otras personas a mí.

\subsubsection{Influencia de los amigos}

La influencia de los amigos también es de gran importancia para esta elección de los estudiantes, influencia que se ha concretado en tres aspectos determinantes: es una salida laboral, aporta a la formación personal y permite tener buen nivel de vida.

[...] algún amigo me ha influenciado y animado a que me embarque en estudiar esta carrera porque me permite seguir formándome como persona, me abre puertas para trabajar y poder tener una vida mejor.

Tabla 2. Matriz de codificación de frecuencias cruzadas entre nodos

\begin{tabular}{llllll}
\cline { 2 - 6 } & $\mathbf{1}$ & $\mathbf{2}$ & $\mathbf{3}$ & $\mathbf{4}$ & $\mathbf{5}$ \\
\hline Influencia de padres y familiares & 5 & 3 & 2 & 1 & 1 \\
\hline Buenas condiciones laborales & 0 & 1 & 0 & 0 & 1 \\
\hline $\begin{array}{l}\text { Influencia del profesorado } \\
\begin{array}{l}\text { Experiencia de trabajo con niños y volun- } \\
\text { tariado }\end{array}\end{array}$ & 4 & 3 & 1 & 0 & 1 \\
\hline Influencia de los amigos & 0 & 1 & 0 & 0 & 1 \\
\hline
\end{tabular}

Nota (1): Elaboración propia. Dimensión vocacional (1). Formar y

educar a los demás (2). Vocación de servicio y altruismo (3). Recompensas morales (4). Interacción con los nińos (5).

Nota (2): Elaboración propia.

\section{Figura 1. Relación entre motivaciones intrínsecas y extrínsecas}

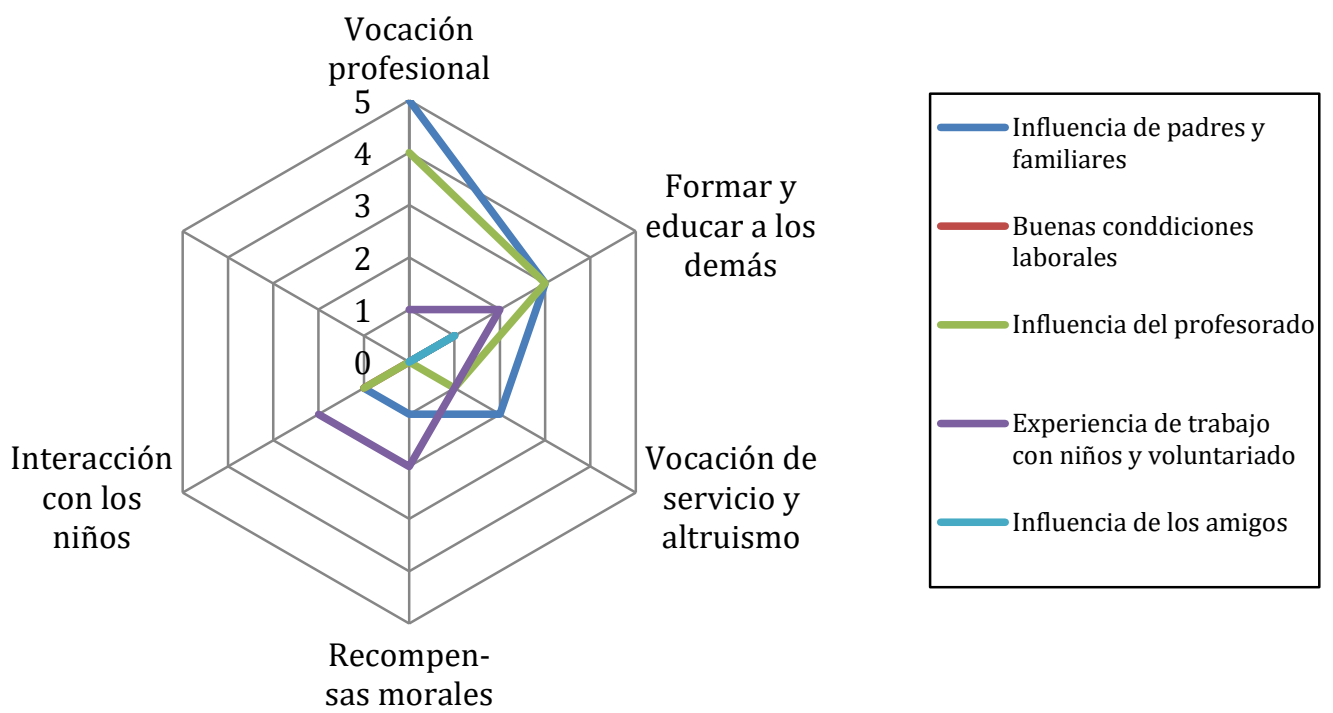

Nota. Elaboración propia, a partir de la matriz de codificación de frecuencias cruzadas entre nodos. 


\section{Discusión y conclusiones}

El análisis de las categorías relativas a las narrativas autobiográficas de los estudiantes plantea diez categorías, cinco son relativas a motivaciones intrínsecas: "dimensión vocacional", "formar y educar a los demás", "vocación de servicio y altruismo", "recompensas morales" e "interacción con los niños", y cinco relativas a motivaciones extrínsecas: "Influencia de los padres", "buenas condiciones laborales", "influencia del profesorado", "experiencia de trabajo con niños y voluntariado", así como "influencia de amigos", observándose que la segunda categoría aducida responde a una motivación extrínseca meramente pragmática.

En una primera reflexión, relativa a las aportaciones que hacen los estudiantes, se observa que las dos dimensiones transversales del discurso de los estudiantes: vocacional y motivacional, aparecen casi siempre en las narrativas de estudiantes con motivación intrínseca y ocasionalmente en el discurso de los que tienen motivaciones extrínsecas. Habitualmente, los que tienen motivaciones extrínsecas solo muestran interés por mejorar la calidad de vida y la estabilidad profesional o la interacción con los niños, con un cierto nivel de empatía hacia ellos, pero sin profundizar en aspectos relativos a la esencialidad del sentido de la vocación (Ramos-Morales \& Serrano-Castañeda, 2017), que evidencia que la realidad esencial de la formación de los estudiantes está inmersa en el idealismo kantiano.

La motivación intrínseca de los estudiantes evidencia una estrecha relación entre vocación y motivación intrínseca, ya que el estudiante, mediante la vocación, conoce en qué consiste su ser y hacia dónde debe orientarse e interaccionar con el mundo (Ayerra, 2016). Es la motivación intrínseca la que permite que se mantengan la concepción vocacional en el tiempo y las disposiciones que la ejercitan, es decir, las denominadas disposiciones hacia la acción decidida (Ossa et al., 2018).

Las categorías definidas se inscriben en una realidad interactiva, ya que coadyuvan al ejercicio de la vocación; así, "formar y educar a los demás" supone una orientación altruista y de compromiso con el "otro" o la "otra", en el sentido de darse uno mismo (Breijo \& Fernández, 2020). Del mismo modo, y aunque con menor incidencia, se evidencia en los estudiantes que una adecuada interacción con los niños tiene como consecuencia recompensas morales en el maestro, mucho más profundas que las recompensas externas, ya que consolidan la vocación profesional (García-Niño, 2019).

Las narrativas autobiográficas ponen de manifiesto la incidencia de las motivaciones extrínsecas en la consolidación de las intrínsecas. A ello se refieren algunos estudiantes al afirmar que "sus padres han sido los que le han trasmitido las sensaciones que se sienten al estar en contacto con los niños en un aula, lo que ha consolidado su convencimiento personal", ejemplo que repercute muy positivamente en la elección de los hijos, ya que la incidencia psicológica, originada por la relación afectiva, así como las evidencias de tipo social y económica, influyen en la elección de la carrera de maestro (Patrón \& Chagoyán, 2019).

El discurso de los estudiantes manifiesta que las motivaciones extrínsecas, determinadas por el logro de recompensas externas, como afirma Del Valle-Uzcátegui (2019), tienen en algunas ocasiones efectos dinamizadores, de carácter psicológico-afectivo, como se ha puesto de manifiesto en la incidencia de los padres-maestros en la elección de la carrera. En otras ocasiones su carácter es contextual, como las referencias a anécdotas divertidas y a la satisfacción de sus familiares. En este segundo caso, también les indujo a elegir la profesión de maestro, pero con menos consistencia (véase Tabla 2 y Figura 1), evidencias que se reflejan en las narrativas mediante aspectos como la trasmisión de motivación y pasión dominante por formar personas y la satisfacción por contribuir al desarrollo del proyecto de vida de niños. Tampoco se profundiza en aquellos aspectos que pueden ser grandes refuerzos morales para mantener viva la ilusión por la formación como actividad profesional (Del Valle-Uzcátegui, 2019; Núñez \& León, 2018).

Las buenas condiciones laborales constituyen la segunda categoría extrínseca en preferencia de los 
estudiantes, ya que suponen la estabilidad laboral y poder acceder a la condición de funcionarios (Cortés et al., 2014; Ferreira-Bolognani \& Mendes-Nacarato, 2015). Quienes así opinan buscan mejor calidad de vida y amplias vacaciones. Así se manifiestan preferentemente los estudiantes de un origen social modesto, que no reflejan motivos consistentes en su elección (Breijo \& Fernández, 2020).

La incidencia de los profesores en las decisiones que toman los informantes respecto a la carrera elegida sigue una pauta similar a la incidencia de los padres. Hay admiración por "la atención que les prestaron", "por lo bien que enseñaban”, "por lo divertidas que hacían las clases”, y eso es importante, pero roza lo anecdótico. No hay ninguna referencia a aspectos humanísticos de la profesión, a la centralidad del maestro como forjador de valores democráticos que va generando hábitos, actitudes y modos de ser en los nińos, o como quien contribuye en primera línea para que los estudiantes conviertan la información en conocimiento; es decir, no hay referencias a la consideración del maestro o maestra como dinamizador de nuevos proyectos de vida. Tan solo un informante alude a su profesor de latín como persona que le enseñó a ver la vida desde distintos puntos de vista (García-Leos, 2020).

Las experiencias de trabajo con niños tienen menos incidencia en el discurso de los estudiantes; estas prácticas se centraron en acciones de voluntariado, atención a los menores en el ámbito familiar y también en los centros educativos, colaboración en comunidades de aprendizaje en tareas de apoyo, mediante grupos interactivos y de aprendizaje dialógico, facilitadores de la comunicación con los niños, que según Vieira-Parra (2010), descubre otras motivaciones intrínsecas.

Los estudiantes que aluden a la influencia de amigos como determinante de su elección exponen en sus autobiografías que los consejos que reciben se refieren a que la profesión de maestro aporta seguridad laboral, la posibilidad de vivir bien (vacaciones), salida profesional y remuneración adecuada. Estas declaraciones suelen darlas los estudiantes que proceden de clase media y media-baja. Sin embargo, los estudiantes de clases sociales más acomodadas aluden a los estudios de Magisterio como una segunda o tercera opción más acorde con sus posibilidades intelectuales; en un caso se observa convergencia de la categoría con las "condiciones laborales" y en otro, una elección determinada por condicionantes externos.

En consonancia con lo anterior, Çermik et al. (2010) encontraron en sus investigaciones una relación positiva entre las preferencias de los estudiantes de niveles socioeconómicos medio y medio-bajo y su visión más pragmática sobre la elección de la carrera.

En las narrativas de los estudiantes de Grado no se observan referencias a la creatividad de los niños, ni a la orientación de la misma. Tampoco se aborda el interés por ayudarles a indagar o a que se planteen preguntas centrales sobre su entorno sociocultural, a nivel de sus posibilidades. Del mismo modo, en las narrativas tampoco se percibe que los futuros maestros vean la formación como proceso previo a la socialización de los niños y niñas en el que deben participar activamente. Los estudiantes que ponen de manifiesto que su trabajo previo con niños les ha sido de gran utilidad no aportan aspectos de gran interés. Si bien es cierto que una alumna afirma que le agradaría dejar su impronta, ningún estudiante expresa inquietudes por que esos chicos adquieran una formación basada en sus potencialidades. Es un contacto muy superficial, del que los futuros estudiantes no transcienden de lo meramente anecdótico.

La percepción que tienen los estudiantes de la profesión de maestro es la de un buen enseñante que se dedica a facilitar los aprendizajes a los alumnos, eso sí, de modo ameno y con sentido ético de su desempeño, pero solamente que transcienda los procesos instructivos. Los estudiantes abordan las motivaciones externas como meras emociones e intereses personales efímeros que, a lo sumo, introducen en las motivaciones internas. No obstante, en ningún momento aluden a aspectos relacionados con la formación integral de la persona. Todo ello lleva a reflexionar sobre las consecuencias de haber optado en la formación por una racionalidad tecnológica como mejor modo de educar, y ello ha contribuido a contemplar 
erróneamente la figura del maestro como tecnólogo en vez de técnico (Mínguez-Vallejos, 2016).

\section{Referencias bibliográficas}

Aisenson, G., Valenzuela, V., Celeiro, R., Bailac, K., \& Legpi, L (2010). El significado del estudio y la motivación escolar de jóvenes que asisten a circuitos educativos diferenciados socioeconómicamente. Psicología Educacional y Orientación Vocacional, 17, 109-119. https://r.issu.edu.do/l?1=10558qAB

Andreu, J., García-Nieto, A., \& Pérez-Corbacho, A. M. (2007). Evolución de la teoría fundamentada como técnica de análisis cualitativo. CIS.

Ayerra, P. (2016). Vocación, conocimiento y libertad en la filosofía de Ortega y Gasset [Trabajo de fin de grado, Universidad de Navarra].

Ballestín, B., \& Fábregues, S. (2018a). Diseño de la investigación cualitativa. En B. Ballestín \& S. Fábregues (Eds.), La práctica de la investigación cualitativa en ciencias sociales y de la educación (pp. 35-94). UOC.

Ballestín, B. (2018b), El análisis de datos cualitativos. En B. Ballestín \& S. Fábregues (Eds.), La práctica de la investigación cualitativa en ciencias sociales y de la educación (pp. 175-247). UOC.

Bernal, C. A. (2016), Metodología de investigación. Administración, economía, humanidades y ciencias sociales. Pearson.

Breijo, T., \& Fernández, M. (2020). En torno al desarrollo de la identidad profesional docente en la formación inicial de los profesores. Mendive, 18(1), 1-4. https://r.issu.edu.do/l?1=10559WHi

Carrero, V., Soriano, R. M., \& Trinidad, A. (2012). Teoria Fundamentada. Grounded Theory. El desarrollo de teoría desde la generalización conceptual. CIS.

Çermik, H., Doğan, B., \& Şahin, A. (2010). Sınıf öğretmenliği öğretmen adaylarının öğretmenlik mesleğini tercih sebepleri. Pamukkale Üniversitesi Eğitim Fakültesi Dergisi, 28(2), 201-212.

http://hdl.handle.net/11499/27706
Clemens, R. F., \& Tierney, W. G. (2020). The uses and usefulness of life history. En M. R. M. Ward \& S. Delamont (Eds.), Handbook of Qualitative Research in Education (pp. 270-284). Edward Elgar Publishing. https://doi.org/10.4337/9781788977159.00034

Cortés, P, Leite, A., \& Rivas, J. I. (2014). Un enfoque narrativo de la identidad profesional en profesorado novel. Tendencias Pedagógicas, 24, 199-214. http://hdl.handle.net/10486/663122

Del Valle-Uzcátegui, Y. (2019). Aspectos motivacionales en la selección de carrera universitaria: un estado del arte. Saber Humano, 9(14), 124-144. https://doi.org/10.18815/sh.2019v9n14.334

Delory-Momberger, C. (2015a). La condición biográfica. Ensayos sobre el relato de si en la modernidad avanzada. Editorial Universidad de Antioquia.

Delory-Momberger, C. (2015b). El relato de sí como hecho antropológico. En G. J. Murillo (Eds.), Narrativas de experiencia en educación y pedagogía de la memoria (pp. 57-68). Editorial Universidad de Antioquia.

Díaz-Barriga, F, López-Banda, E. A., \& Vázquez-Negrete, V. (2018). Exploración de los fondos de identidad en estudiantes de posgrado mediante una adaptación de la Multimetodología autobiográfica extendida (MAE). Papeles de Trabajo sobre Cultura, Educación y Desarrollo Humano, 14(1), 1-22. https://r.issu.edu.do/l?l=10560g3a

Dubard, C., \& Nicourd, S. (2017). Les biographies en sociologie. Éditions La Découverte.

Egido, I. (2010). El acceso a la profesión docente en España en perspectiva europea. Algunas reflexiones orientadas a la mejora de la selección del profesorado. Educación XX1, 13(2), 47-67.

https://doi.org/10.5944/educxx1.13.2.23

Ferreira-Bolognani, M. S., \& Mendes-Nacarato, A. (2015). Las narrativas de vida como prácticas de (auto) formación de maestras que enseñan matemáticas. Revista Mexicana de Investigación Educativa, 20(64), 171-193. http://ref.scielo.org/qc249z 


\section{Revista Caribeña de Investigación Educativa | 2021, 5(2), 38-51}

Feu, S., Vizueta, M., De la Cruz, E. \& Gragera, A. (2016). Importancia de las experiencias previas en la vocación y elección de las titulaciones de maestro con mención en Educación Física. Movimiento. Revista de Educação Física da UFRGS, 22(3), 929-942. https://doi.org/10.22456/1982-8918.59282

Flick, U. (2012). Introducción a la investigación cualitativa. Morata.

Leos, J. L. G. (2020). Desarrollo profesional docente: relatos autobiográficos de maestros en servicio. Debates por la Historia, 8(2), 255-269.

https://r.issu.edu.do/l?1=10288fhq

García-Niño, G. (2019). Percepción y satisfacción de los docentes sobre la acción tutorial en las instituciones educativas "Sechura". [Tesis de maestría en Educación, Universidad de Piura]. PIRHUA.

https://pirhua.udep.edu.pe/handle/11042/4326

Gómez-Acuñas, M. M. (2003). Variables de éxito en la formación del profesorado [Tesis doctoral, Universidad de Extremadura].

http://dehesa.unex.es/handle/10662/513

González, M.F. \& Padilla, M. T. (2014), Investigación narrativa: las historias de vida. En B. Ballesteros (Coord.), Taller de investigación cualitativa (pp.77101). UNED.

Gratacós, G. (2014). Estudios sobre la motivación en la elección de ser maestro. Servicio de publicaciones de la Universidad Internacional de Catalunya.

Jarauta-Barranco, B. (2017). La construcción de la identidad profesional del maestro de primaria durante su formación inicial. El caso de la Universidad de Barcelona. Profesorado. Revista de Curriculum y Formación del Profesorado, 21(1), 103-122.

http://hdl.handle.net/2445/122650

Leite, A. E., Rivas, J. I., \& Cortés, P. (2019), Narrativas, enseñanza y universidad. En R. B. Martín, M. C. Rinaudo \& P. V. Paoloni (Eds.), Comunidades: Estudios y experiencias sobre contextos y comunidades de aprendizaje (pp. 61-73). Eduvim.
Mínguez-Vallejos, R. (2016). Sobre el modo de ser maestro: una reflexión pedagógica. En: I. Carrillo (Ed.), Democracia y educación en la formación docente (pp. 247-251). Servicio de Publicaciones de la UVic-UCC.

Mínguez-Vallejos, R. (2019). Presentación: La educación ante los retos de una nueva ciudadanía: implicaciones éticas. Educatio Siglo XXI, 37(1), 11-20. https://r.issu.edu.do/l?l=10289DYU

Moriña, A. (2017). Investigar con historias de vida. Metodología biográfico-narrativa. Narcea.

Murcio, A. (2013). Interpretar. De la comprensión previa a la explicación de los acontecimientos. Narcea.

Núńez, J., \& León, J. (2018). Probando las relaciones entre la motivación global, contextual y situacional: un estudio longitudinal de los efectos horizontal, arriba-abajo y abajo-arriba. Revista de Psicodidáctica, 23(1), 9-16.

https://doi.org/10.1016/j.psicod.2017.07.003

Orbegoso, A. (2016). La motivación intrínseca según Ryan \& Deci y algunas recomendaciones para maestros. Educare, Revista Cientifica de Educação, 2(1), 75-93.

http://dx.doi.org/10.19141/2447-5432/lumen.v2.n1.p.75-93

Ossa, C. J., Gromiria, N., Palma, M. R., Arteaga, I., \& Quintana, M. (2018). Construcción y análisis psicométrico del Cuestionario de Vocación Docente para estudiantes (CVD-E). Revista de Estudios y Experiencias en Educación, 17(34), 15-29.

http://doi.org/10.21703/rexe.20181734cossa6

Packer, M. (2018), La ciencia de la investigación cualitativa. Ediciones Uniandes.

Patrón, A., \& Chagoyán, P. (2019). Los inicios de la identidad profesional en la formación del profesorado de secundaria en Guanajuato, México, Profesorado. Revista de Currículum y Formación del Profesorado, 23(3).

http://doi.org/10.30827/profesorado.v23i3.9399 


\section{PÉREZ-FERRA • MARTOS-ORTEGA • QUIJANO-LÓPEZ • GARCÍA-MARTÍNEZ}

Explorando las motivaciones de los futuros docentes de educación primaria en la elección...

Ramos-Morales, J. M., \& Serrano-Casteñeda, J. A. (2017). A escrita autobiográfica de jovens e adultos no ensino superior. Revista Brasileira do Educação de Jovens e Adultos, 5(10), 77-89. https://rissu.edu.do/l?l=102904dd

Rosenthal, G. (2018), Interpretive Social Research. An Introduction. Göttingen University Press. https://doi.org/10.17875/gup2018-1103

Runge, A. K., \& Muñoz, D. A. (2015). Los docentes y la tematización de sí: formación y narración de sí en clave antropocrítica. En G. J. Murillo (Ed.), $N a$ rrativas de experiencia en educación y pedagogía de la memoria (pp. 215-235). FFyL-UBA/CLACSO/Universidad de Antioquia.
Thomas, J., \& Harden, A. (2008). Methods for the Thematic Synthesis of Qualitative Research in Systematic Review. BMC Medical Research Methodology, 8(45), 1-10. https://doi. org/10.1186/1471-2288-8-45

Vieira-Parra, L. (2010). Voluntariado en la escuela. Un estudio de casos dentro del proyecto comunidades de aprendizaje [Tesis de doctorado, Universidad de Barcelona].

Watson, C., \& Mcluckie, C. (2020), Analysing narratives: the narrative construction of professional identity. En M. R. M. Ward \& S. Delamont (Eds.), Handbook of Qualitative Research in Education (pp. 380-391). Edward Elgar Publishing. https://doi. org/10.4337/9781788977159.00045

\section{CÓMO CITAR:}

Pérez-Ferra, M., Martos-Ortega, Quijano-López, R., J. M., \& García-Martínez, I. (2021). Explorando las motivaciones de los futuros docentes de educación primaria en la elección de sus estudios. RECIE. Revista Caribeña de Investigación Educativa, 5(2), 38-51. https://doi.org/10.32541/recie.2021.v5i2.pp38-51 